\title{
Traffic Signal as a use case of Python with Raspberry Pi
}

\author{
Nanasaheb M. Halgare, PhD \\ CSE, Assistant Professor \\ M.S. Bidve Engineering College \\ Latur
}

\begin{abstract}
As we know that there are many resources and technologies are launched now a days in computer society like R+,Jquery ,etc and all these languages are execute on Computers as well as but it is quiet hard to maintain the computer and laptop for the students because of its higher weight, larger size, high cost and to overcome all these factors Creator Eben Upton's launch a device which is comparatively small in size, low in cost, and lesser in weight callas Raspberry pi.in this paper we are dealing with what is raspberi pi and how python programming works with raspberry pi..
\end{abstract}

\section{General Terms}

language,LED et. al.

\section{Keywords}

Raspberry pi ,Python.

\section{INTRODUCTION}

A Raspberry $\mathrm{Pi}$ is a debit card-sized computer newly designed for education, encouraged by the 1981 BBC Micro. Creator Eben Upton's aim was to launch a low-cost device that would enhance programming skills and hardware understanding at the pre-university level. But thanks to its small size and affordable price, it was rapidly accepted by tinkerers, makers, and electronics enthusiasts for projects that need more than a basic microcontroller (such as Arduino devices).The Raspberry Pi is slower than a modern laptop or desktop but is still a complete Linux computer and can accommodate all the expected capabilities that implies, at a dipressed-power consumption level.Python is an easy to learn, competent programming language. It has efficient high-level data structures and a simple but powerfull approach to objectoriented programming. Python's.

\section{RASPBERRY PI OPEN HARDWARE}

The Raspberry Pi is nothing but a open hardware, with the passing over of the primary chip on the Raspberry $\mathrm{Pi}$, the Broadcomm SoC (System on a Chip), which executes many components of the board-CPU, like graphics, memory, the USB controller, etc. Number of the projects built with a Raspberry Pi are open and well-documented as well and are things you can build and modify easily.

\section{WHAT ARE THE DIFFERENCES IN RASPBERRY PI MODELS?}

The Raspberry Pi launched recently a new model, the Raspberry Pi 2, which replaces some of the previous boards, even if the earlier boards will still be formed as long as there is a call for them. It is generally rearward adaptable with previous versions of the board, so any projects assignment you see which were built for a earlier version of the board should still work. There are a two Raspberry Pi models, the A and the B, named after the aforementioned BBC Micro, which was also released in a Model A and a Model B. The A comes with $256 \mathrm{MB}$ of RAM and one USB port. It is cheaper and uses less power than the $\mathrm{B}$. The current model $\mathrm{B}$ comes with a second USB port, an Ethernet port for connection to a network, and 512MB of RAM.The Raspberry Pi A and B boards been upgraded to the $\mathrm{A}+$ and $\mathrm{B}+$ respectively.

\section{WHAT KIND OF OPERATING SYSTEM DOES THE RASPBERRY PI RUN?}

Basically The Raspberry Pi was constructed for the Linux operating system, and many Linux dispersion have a version optimized for the Raspberry Pi.There are Two options first one is Raspbian, which is based on the Debian operating system, and second one is Pidora, which is based on the Fedora operating system. For newcomers, either of these two work well.

\section{WHAT IS PYTHON LANGUAGE}

Python is an explained, object-oriented, high-level programming language with dynamic meaning. Its high-level in built data structures, combined with dynamic typing and dynamic binding, make it very beautiful for fast Application Development, as well as for use as a scripting or glue language to bridge existing components together. Python's lucid, royal to learn syntax highlight creditability and therefore curtail the cost of program maintenance. Python guide modules and packages, which boost program structure and code reuse. The Python interpreter and the extensive standard library are available in source or binary form without charge for all major platforms, and can be freely distributed.

Because of no compilation step, the edit-test-debug cycle is fast. Debugging Python programs is easy: a bug or bad input will never cause a segmentation fault. Instead, when the interpreter discovers an error, it raises an exception. When the program doesn't catch the exception, the interpreter prints a stack trace. A source level debugger allows inspection of local and global variables, evaluation of arbitrary expressions, setting breakpoints, stepping through the code a line at a time, and so on. The debugger is written in Python itself, testifying to Python's introspective power. On the other hand, often the quickest way to debug a program is to add a few print statements to the source: the fast edit-test-debug cycle makes this simple approach very effective. 


\section{SMART TRAFFIC SYSTEM USING RASPBERRY PI AND PYTHON}

To implement such system there is need to see working of GPIO

\begin{tabular}{|c|c|c|c|c|c|}
\hline GPIO.BCM & Function & GPIO. & SOARD & Function & GPIO.BCM \\
\hline$\angle 50 \mathrm{~mA}$ & $3 \sqrt{3}$ & 1 & 2 & $5 \mathrm{~V}$ & \\
\hline BCM GPIO02 & SDA1 ARM & 3 & 4 & $5 \mathrm{~V}$ & \\
\hline BCM GPIO03 & SCL1 ARM & 5 & 6 & GND & \\
\hline \multirow[t]{2}{*}{ BCM GPIO04 } & & 7 & 8 & $\mathrm{TX}$ & BCM GPIO14 \\
\hline & GND & 9 & 10 & $\mathrm{RX}$ & BCM GPIO15 \\
\hline BCM GPIO17 & SPI1 CE1 & 11 & 12 & PWMO/SPI1 CEO & BCM GPIO18 \\
\hline BCM GPIO27 & & 13 & 14 & GND & \\
\hline BCM GPIO22 & & 15 & 16 & & BCM GPIO23 \\
\hline$<50 \mathrm{~mA}$ & $3 \times 3$ & 17 & 18 & & BCM GPIO24 \\
\hline BCM GPIO10 & SPIO MOSI & 19 & 20 & GND & \\
\hline BCM GPIO9 & SPIO MISO & 21 & 22 & & BCM GPIO25 \\
\hline \multirow[t]{2}{*}{ BCM GPIO11 } & SPIO SCLK & 23 & 24 & SPIOCEO & BCM GPIO08 \\
\hline & GND & 25 & 26 & SPIOCE1 & BCM GPIO07 \\
\hline BCM GPIO00 & SDAOVC & 27 & 28 & SCLOVC & BCM GPIO01 \\
\hline BCM GPIO05 & & 29 & 30 & GND & \\
\hline BCM GPIO06 & & 31 & 32 & PWMO & BCM GPIO 12 \\
\hline BCM GPIO13 & PWM1 & 33 & 34 & GND & \\
\hline BCM GPIO19 & SPI1 MISO/PWM1 & 35 & 36 & SPI1 CE2 & BCM GPIO16 \\
\hline \multirow[t]{2}{*}{ BCM GPIO26 } & & 37 & 38 & SPI1 MOSI & BCM GPIO2O \\
\hline & GND & 39 & 40 & SPI1 SCLK & BCM GPIO21 \\
\hline
\end{tabular}

\section{1 .Hardware}

As well as a Raspberry Pi with an SD card and the usal peripherals, you'll also need:

- $\quad 1 x$ Solderless breadboard

- $\quad$ All king of jumper leads

- $1 x$ Tactile button

- 3x LEDs (Red,Greean and Yellow)

- 3 x 330 ohm Register

- $\quad$ Buzzer

- Button

\subsection{Software}

Raspberry Pi require NOOBS OS. It is a way of making setting up a Raspberry Pi for the first time.

\subsection{GPIO pins}

One competent feature of the Raspberry Pi is the row of GPIO pins along the top edge of the boadr. GPIO stands for General-Purpose Input/Output. These pins are a physical interface between the Raspberry Pi and the outside world.
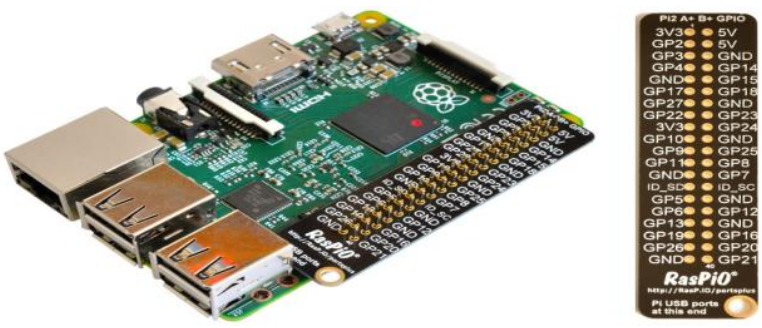

Fig 6.1

If don't have a pin label, then this can help you to identify the pin numbers .

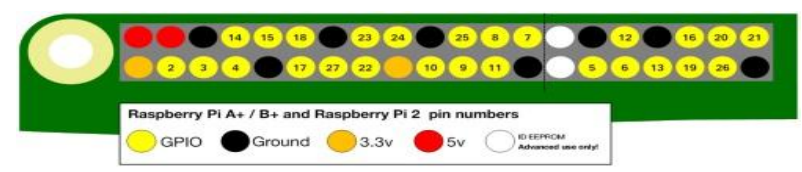

Fig 6.2
3.3 volts

Everything connected to these pins will always get $3.3 \mathrm{v}$ of power.

5 volts

Everything connected to these pins will always get $5 \mathrm{v}$ of power.

Gnd

Zero volts, used to complete a circuit

\section{GPIO}

These pins are used for general-purpose use and can be configures as input or output pins

\section{ID_SC/ID_SD/DNC}

Special purpose pins.

\section{Lighting an LED}

LEDs are delicate little things. if you put too much current through them they will pop. To limit the current going through the LED, you should always use a register in series with it.

Long leg an LED to the Pi $3.3 \mathrm{v}$ and the short leg to a GND pin. Automatically LED will turn ON

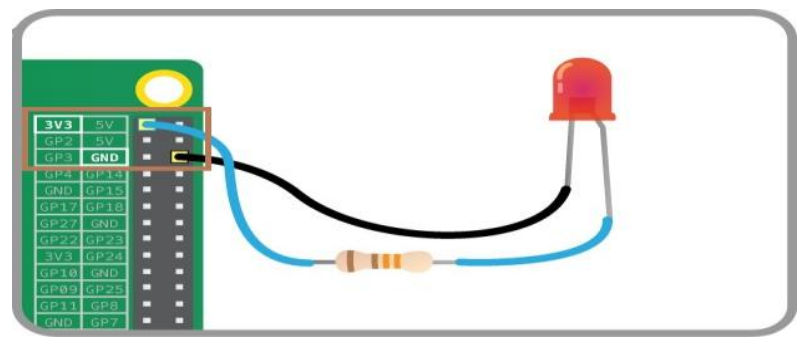

Fig 6.3

If you connect special purpose(GPIO) pin are connect to long leg, make sure to write the import code.

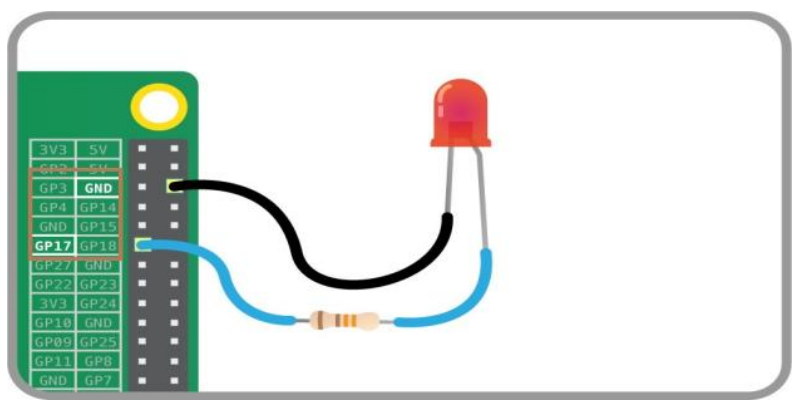

Fig 6.4

Switching an LED on and off

GPIO Zero is a new Python library which provides a simple interface to everyday GPIO component. It comes installed by defalut in Rasbian.

Open IDLE(Integrated Development Environment), which you can use to write and run code.

Raspbian Menu Icon >> Programming >> Python 3 (IDLE). 


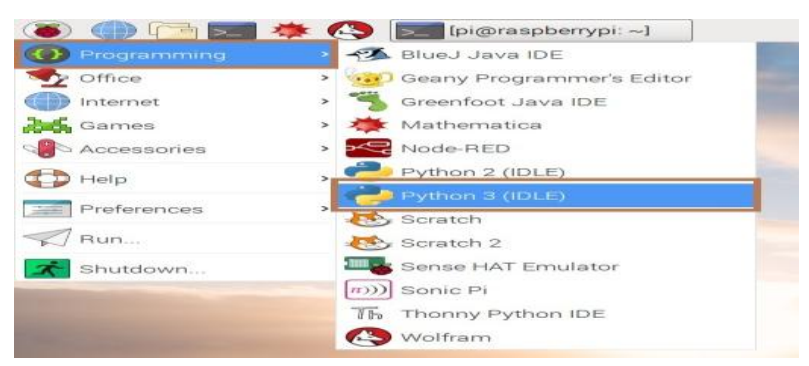

Fig 6.5

- To create a new file in IDLE, You can click on File and then New File in IDLE's menu bar.

- Create a new file by clicking File >> New File

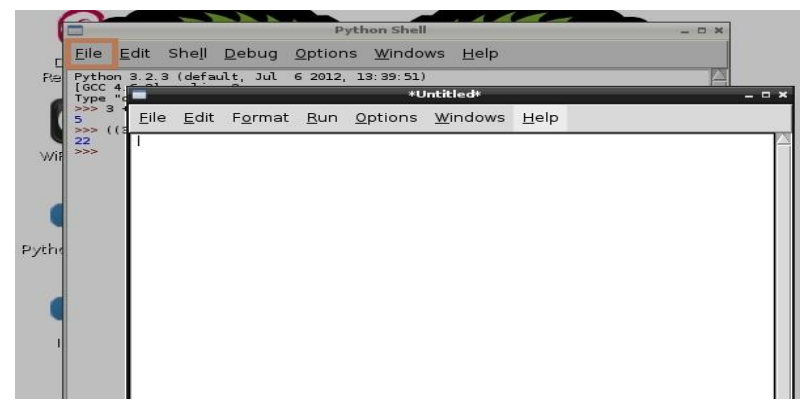

Fig 6.6

- $\quad$ Save the new file by clicking File $>>$ Save. Save the file as trffic.py

- You'll need the LED Class, and to tell it that the LED is on pin 17. Write the following code in your new file.

- from gpiozero import LED

- $\quad$ led $=\operatorname{LED}(17)$

- To make the LED switch on, type the following and press Enter

- led.on()

- To make it swith off you can type

- led.off()

Your LED should switch on and then off again. But that's not all you can do. Similary check the Buzzer and Button. Just import a Buzzer and Button for header file.

\section{Making Traffic Light}

We need a breadboard, three LEDs, a button, a buzzer and the necessary jumper cables and registors.

\section{Wiring}

First, we need to understand how each component is connected.

- A push button requires 1 ground pin and 1 GPIO pin

- An LED requires 1 ground pin and 1 GPIO pin, with a current limiting registor

- A buzzer requies 1 ground pin and 1 GPIO pin

Place the components on the breadboard and connect them to the Raspberry Pi GPIO pins, accoring to the following diagram.

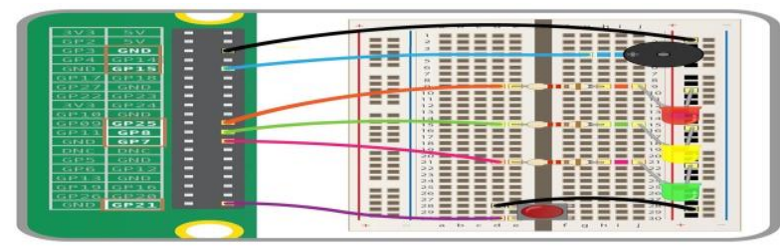

Fig 6.7

Component GPIO pin

- $\quad$ Button21

- $\quad$ Red LED25

- $\quad$ Yellow LED8

- $\quad$ Green LED7

- $\quad$ Buzzer15

This is the same as the Switching and LED on and off step

- Open Pyton 3 from the main menu

- Create a new file just save with the project name.py

Add TrafficLight, Button and Buzzer Code

- from gpiozero import Button, TrafficLights, Buzzer

- $\quad$ from time import sleep

- $\quad$ buzzer $=\operatorname{Buzzer}(15)$

- $\quad$ button $=$ Button $(21)$

- $\quad$ lights $=$ TrafficLights $(25,8,7)$

- while True:

- $\quad$ button.wait_for_press()

- $\quad$ buzzer.on()

- light.green.on()

- $\quad \operatorname{sleep}(1)$

- $\quad$ lights.amber.on()

- $\quad \operatorname{sleep}(1)$

- lights.red.on()

- $\quad \operatorname{sleep}(1)$

- $\quad$ lights.off()

- $\quad$ buzzer.off() 


\section{RESULT}

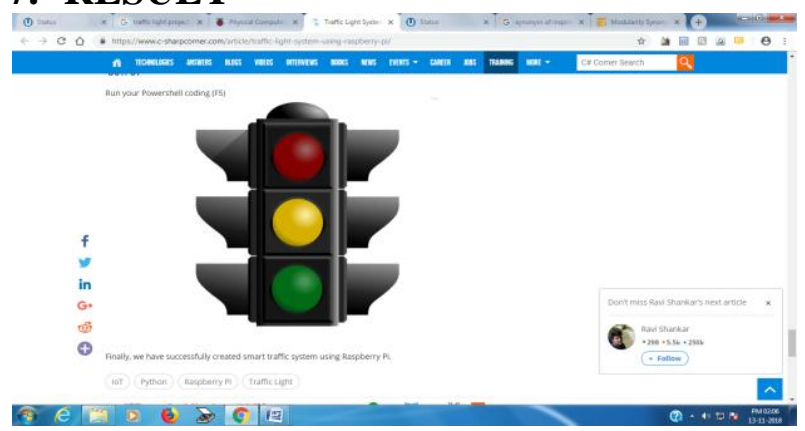

Fig 7 yellow light glowing

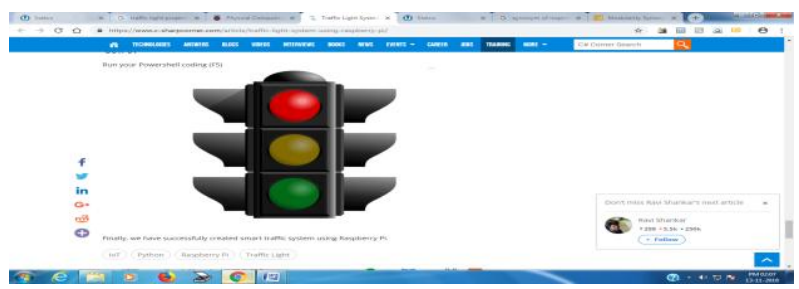

Fig 7.1 red light glowing

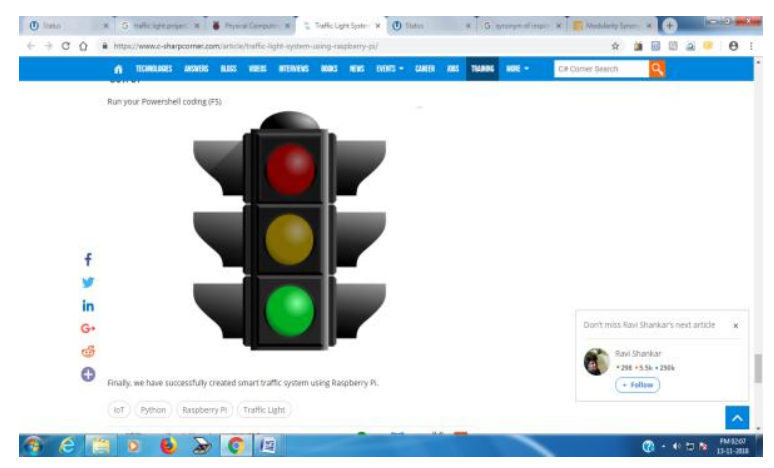

Fig 7.2green light glowing

\section{CONCLUSION}

Raspberry pi and python is recently launched resource and technology and in this paper we implemented traffic light system using Raspberry pi and python as on of of the use case.

\section{REFERENCES}

[1] https://www.raspberrypi.org/

[2] Python.org.

[3] http://www.engpaper.com/raspberry-pi-2018 